\title{
Prognostic Significance of Troponin Elevation for Long-Term Mortality after Ischemic Stroke
}

\author{
Sung-Ho Ahn, ${ }^{\text {a,b }}$ Ji-Sung Lee, ${ }^{\mathrm{c}}$ Young-Hak Kim, ${ }^{\mathrm{d}}$ Bum Joon Kim, ${ }^{\mathrm{e}}$ Yeon-Jung Kim, ${ }^{\mathrm{a}}$ Dong-Wha Kang, \\ Jong S. Kim, ${ }^{\text {a }}$ Sun U. Kwon ${ }^{\text {a }}$ \\ ${ }^{a}$ Department of Neurology, Asan Medical Center, University of Ulsan, College of Medicine, Seoul, Korea \\ ${ }^{b}$ Department of Neurology, Research Institute for Convergence of Biomedical Science and Technology, Pusan National University Yangsan \\ Hospital, Pusan National University School of Medicine, Busan, Korea \\ 'Clinical Research Center, Asan Medical Center, University of Ulsan, College of Medicine, Seoul, Korea \\ ${ }^{\mathrm{d}}$ Division of Cardiology, Asan Medical Center, University of Ulsan, College of Medicine, Seoul, Korea \\ eDepartment of Neurology, Kyung Hee University Medical Center, Kyung Hee University School of Medicine, Seoul, Korea
}

Background and Purpose Troponin, a marker of myocardial injury, frequently increases and is related with poor outcome in ischemic stroke patients. However, the long-term outcome of this elevation remains uncertain. We, therefore, investigated the prognostic significance of troponin elevation for long-term mortality, and explored factors affecting troponin elevation after ischemic stroke.

Methods We retrospectively analyzed the medical data of stroke patients who were admitted within 24 hours of symptom onset and underwent a serum cardiac troponin I test at admission during a five-year period. Information on mortality as the outcome was obtained from the National Death Certificate system.

Results A total of 1,692 patients were eligible for inclusion with 33 months of median follow-up. Troponin elevation that exceeded the 99th percentile $(>0.04 \mathrm{ng} / \mathrm{mL}$ ) of values was detected in 166 patients $(9.8 \%)$. After adjusting for baseline characteristics, troponin elevation was associated with previous ischemic heart disease and congestive heart failure, comorbid atrial fibrillation and active cancer, and increased National Institutes of Health Stroke Scale score. Patients with troponin elevation had a high risk of overall death (hazard ratio [HR] 1.83, 95\% confidence interval $[\mathrm{Cl}] 1.40-2.40$ ), including stroke-related (HR 1.71,95\% Cl 1.14-2.55), cardiac-related (HR 3.17, 95\% Cl 1.49-6.74), and cancer-related ( $\mathrm{HR} 1.98,95 \% \mathrm{Cl} 1.14-3.45)$ death than those without troponin elevation.

Conclusions Troponin elevation in the acute stage of ischemic stroke was associated with long-term mortality, mainly due to increased stroke- and cancer-related death in the first year and cardiacrelated death in the later period.

Keywords Stroke; Prognosis; Troponin
Correspondence: Sun U. Kwon Department of Neurology, Asan Medical Center, University of Ulsan College of Medicine, 88 Olympic-ro 43-gil, Songpa-gu, Seoul 05505, Korea

Tel: +82-2-3010-3960

Fax: +82-2-474-4691

E-mail:sunkwon7@gmail.com

Received: December 7, 2016

Revised: April 12, 2017

Accepted: May 3, 2017

The authors have no financial conflicts of interest.

\section{Introduction}

Cardiac troponins are sensitive and specific biomarkers of cardiac injury; thus, elevated level of troponin is widely used for the diagnosis of acute myocardial infarction, ${ }^{1}$ risk stratification of future cardiovascular events for patients with coronary disease or stable angina, and even in apparently healthy individuals. $^{2}$ Furthermore, currently, troponin elevation has also been frequently observed in patients with ischemic stroke ${ }^{3,4}$ and related with increased risk of death or disability and both in 
these patients. $^{5}$

However, the pathomechanism of troponin elevation in ischemic stroke patients is still controversial, whether it is due to the concomitant cardiac ${ }^{3,6}$ or neurological ${ }^{7,8}$ problems or others. ${ }^{9}$ In addition, the evaluation of clinical impact of troponin elevation was limited to a short-term period within 1 year after stroke; thus, the prognostic value of troponin elevation in ischemic stroke patients for a long-term survival has not been fully explained.

Therefore, we aimed to assess the determinants of troponin elevation and investigate the long-term mortality and cause of death on the basis of the elevation of cardiac troponin I in patients at the acute stage of ischemic stroke. In addition, we reconfirmed the long-term prognostic value of troponin elevation using a different cut-off point for the definition of troponin elevation based on tertiles of a detectable troponin I instead of the standard cut-off point (e.g., the 99th percentile of troponin) and in subgroups according to the presumable etiologies of ischemic stroke.

\section{Methods}

\section{Study population}

We conducted a retrospective analysis of prospectively registered patients with acute stage of ischemic stroke who were consecutively admitted to Asan Medical Center within 24 hours of symptom onset between May 2007 and December 2011 in electronic medical record. All patients underwent cardiac enzyme testing, including troponin I level, and a 12-lead electrocardiographic (ECG) investigation at admission according to the stroke study protocols in our center, which abide by the guidelines from 2007. ${ }^{10}$ Patients underwent additional cardiac evaluations by a cardiologist if they were suspected acute coronary syndrome during the emergency department evaluation. After admission to the stroke center, patients were excluded if 1) they were diagnosed with acute coronary syndrome during hospital stay; or 2) having insufficient clinical, laborato$r y$, or ECG data.

Clinical data, including demographic characteristics, conventional risk factors for stroke, comorbidities, reperfusion therapy, and the National Institutes of Health Stroke Scale (NIHSS) ${ }^{11}$ were obtained from the registered data. Comorbidities included previous history of stroke, ischemic heart disease (IHD; comprising history of acute myocardial infarction, angina, percutaneous coronary intervention and coronary artery bypass grafting) and congestive heart failure (CHF; defined as having a history of cardinal manifestations and treatment for heart failure), and concomitant atrial fibrillation (AF; known medical history of $A F$, evidence of AF on admission 12-lead ECG and newly diagnosed AF after admission), renal insufficiency (estimated glomerular filtration rate $<60 \mathrm{~mL} / \mathrm{min} / 1.73 \mathrm{~m}^{2}$ at admission) and active cancer (cancer within 6 months prior to enrollment, any treatment for cancer within the previous 6 months, or recurrent or metastatic cancer). Reperfusion therapy was defined as intravenous tissue plasminogen activator or intra-arterial reperfusion therapy and both. ${ }^{12}$ The pathogenesis of ischemic stroke was classified according to the Trial of Org 10172 in Acute Stroke Treatment (TOAST) classification. ${ }^{13}$ The Institutional Review Board of the Asan Medical Center approved this study.

\section{Cardiac investigation}

The lower limit of detection of serum cardiac troponin I (Abbott Laboratories, Abbott Park, IL, USA: 3rd generation troponin I assay [i.e., sensitive contemporary assay]) was $0.006 \mathrm{ng} / \mathrm{mL}$, and the 99th percentile of the upper reference limit of this study was $0.04 \mathrm{ng} / \mathrm{mL}$. Hence, significant elevation of troponin was defined as $>0.04 \mathrm{ng} / \mathrm{mL}$ based on the 99th percentile rule as a standard cut-off point. In addition, troponin elevation was differently defined as above the $3 \mathrm{rd}$ or 2 nd tertile of a detectable troponin I ( $>0.006 \mathrm{ng} / \mathrm{mL})$ to reconfirm the long-term prognostic value of troponin elevation with an increased sensitivity and predictive power. A 12-lead ECG was recorded in all patients at admission and processed using the Marquette 12SL ECG Analysis Program (GE Healthcare, Waukesha, WI, USA). The resultant 12-lead ECG waveforms were uploaded in digital form and interpreted by a cardiologist according to a modified version of the Minnesota code. ${ }^{14}$

Two-dimensional transthoracic echocardiography was conducted in patients with cardiac diseases and suspected of cardiogenic embolic or unknown etiologies of stroke. Wall motion abnormality (WMA) was defined as wall motion score index $>1$ using a standard 16-segment model. Hypertrophic myocardium (HM) was defined as left ventricular mass index $>95 \mathrm{~g} / \mathrm{m}^{2}$ for women and $>115 \mathrm{~g} / \mathrm{m}^{2}$ for men. Reduced ejection fraction (EF) was defined as $<50 \%$. ${ }^{15,16}$

\section{Collection of mortality data}

Follow-up information for patients was obtained using the $\mathrm{Na}$ tional Death Certificate data from the Korean National Statistical Office until December 31, 2012. The official nationwide data for death certificates produced by the Korean National Statistical Office are updated annually and have been used in previous studies as a reliable data source. Deaths were classified according to the International Classification of Diseases 
(ICD), Tenth Revision. ${ }^{17}$ The causes of death were classified as stroke death (ICD code: 160-I69), cardiac cause (ICD code: I20-I25 or I30-I52), malignancies (ICD code: C00-C96), and other causes.

\section{Statistical analysis}

The characteristics of study subjects (Table 1) were compared between those with troponin elevation and without troponin elevation. For the comparisons, Pearson's chi-square test, Fisher's exact test, Student's t-test, and Wilcoxon rank sum test were used according to the types of variables, as appropriate. To analyze the factors affecting troponin elevation, variables in Table 1 (except stroke subtype) were included as potential factors in the multivariable logistic regression. Cumulative incidence rates of mortality were estimated by the Kaplan-Meier method and compared by the log-rank test. To reduce the effect of selection bias and potential confounding, we adjusted for differences in baseline characteristics by weighted Cox pro- portional-hazards regression models and inverse-probability-of-treatment weighting (IPTW). ${ }^{18}$ With this technique, weights for patients with troponin elevation were the inverse of the 1-propensity score, and weights for patients without troponin elevation were the inverse of the propensity score. When applying the IPTW method, a stabilized weight was used to maintain the same number of subjects used in the weighted analysis as the number of original study subjects and to yield more precise interval estimates closer to 95\% coverage probabilities. ${ }^{19,20}$ The discrimination and calibration abilities of the propensity score model were assessed using $\mathrm{C}$ statistics and the Hosmer-Lemeshow test. The model was fairly calibrated ( $P=0.066$ by Hosmer-Lemeshow test) with reasonable discrimination (C statistics $=0.696$ ) (Supplementary Table 1). In addition to reduce residual confounding, we investigated the predictive value of troponin elevation for all-cause and cause-specific mortality in different etiological subgroups (e.g., cardioembolic and non-cardioembolic stroke subtype) after adjusting for

Table 1. Characteristics of the study groups

\begin{tabular}{|c|c|c|c|}
\hline \multirow{2}{*}{ Variable } & \multicolumn{2}{|c|}{ Cardiac troponin I } & \multirow{2}{*}{$P^{*}$} \\
\hline & Elevated $(n=166)$ & Non-elevated $(n=1,526)$ & \\
\hline Age (years) & $68.5 \pm 13.3$ & $65.7 \pm 12.3$ & 0.01 \\
\hline Male & $97(58.4)$ & $937(61.4)$ & 0.46 \\
\hline Heart rate (beats a minute) & $80.6 \pm 20.2$ & $77.3 \pm 17.6$ & 0.03 \\
\hline \multicolumn{4}{|l|}{ Medical history } \\
\hline Hypertension & $112(67.5)$ & 964 (63.2) & 0.27 \\
\hline Diabetes mellitus & $37(22.3)$ & 391 (25.6) & 0.35 \\
\hline Hyperlipidemia & $34(20.5)$ & $352(23.1)$ & 0.45 \\
\hline Current smoking & $44(26.5)$ & $474(31.1)$ & 0.23 \\
\hline \multicolumn{4}{|l|}{ Comorbidities } \\
\hline Previous stroke & $43(25.9)$ & $396(26.0)$ & 0.99 \\
\hline Previous ischemic heart disease & $38(22.9)$ & $194(12.7)$ & $<0.01$ \\
\hline Previous congestive heart failure & $35(21.1)$ & $142(9.3)$ & $<0.01$ \\
\hline Atrial fibrillation & $70(42.2)$ & 409 (26.8) & $<0.01$ \\
\hline Renal insufficiency & $34(20.5)$ & $193(12.6)$ & 0.01 \\
\hline Active cancer & $26(15.7)$ & $63(4.1)$ & $<0.01$ \\
\hline Reperfusion therapy & $19(11.4)$ & $186(12.2)$ & 0.78 \\
\hline NIHSS score & $7[3,14]$ & $4[2,9]$ & $<0.01$ \\
\hline Stroke subtypes & & & $<0.01$ \\
\hline Large artery atherosclerosis & 31 (18.7) & $483(31.7)$ & \\
\hline Cardiogenic embolism & $74(44.6)$ & $420(27.5)$ & \\
\hline Small vessel disease & $9(5.4)$ & 361 (23.7) & \\
\hline Undetermined etiology & $27(16.3)$ & $199(13.0)$ & \\
\hline Other etiology & $25(15.1)$ & $62(4.1)$ & \\
\hline
\end{tabular}

Variables are presented as mean \pm standard deviation, median [interquartile range], or number (\%).

NIHSS, National Institutes of Health stroke scale.

* P-values are calculated by Pearson chi-square test, Fisher's exact test, Student's t-test and Wilcoxon rank sum test as appropriate. 
baseline characteristics, with the additional inclusion of C-reactive protein (CRP) level by weighted Cox proportional hazards regression models. All reported $P$-values were two-sided, and a $P<0.05$ was considered statistically significant. SAS software, version 9.4 (SAS Institute Inc., Cary, NC, USA), was used for statistical analyses.

\section{Results}

\section{Baseline characteristics}

Among 1,823 patients who were admitted to the stroke center with acute ischemic stroke within 24 hours of symptom onset, 131 patients were excluded due to the diagnosis of acute coronary syndrome $(n=18)$ during hospitalization at the stroke center and insufficient quality of clinical, laboratory, or ECG data $(n=113)$. Finally, 1,692 patients were eligible for the analysis. The mean age was $66.0 \pm 12.4$ years (range, $24-96$ years), and 1,034 patients $(61.1 \%)$ were male patients. The range of serum cardiac troponin I values in all patients was $0.057 \pm 0.699 \mathrm{ng} / \mathrm{mL}$ (mean \pm standard deviation [SD]) with an extremely skewed distribution to the right side because 950 patients (56.3\%) had a minimum level of troponin I $(0.006 \mathrm{ng} /$ $\mathrm{mL}$ ) and 742 patients (43.7\%) had a wide range of troponin I level (from 0.007 to $25.10 \mathrm{ng} / \mathrm{mL}$ ). Among 742 patients who had a detectable troponin I $(>0.006 \mathrm{ng} / \mathrm{mL})$, the $3 \mathrm{rd}$ and $2 \mathrm{nd}$ tertile of a detectable troponin was a $0.025 \mathrm{ng} / \mathrm{mL}$ and 0.012 $\mathrm{ng} / \mathrm{mL}$ respectively (Figure 1 ).

Troponin elevation was identified in 166 patients (9.8\%) patients, and the mean duration of time interval from the symptom onset to admission $\left(7.7 \pm 6.6\right.$ hours $\left[m_{e a n} \pm S D\right]$ vs. $8.4 \pm 7.0$ hours [mean $\pm S D], P$-value $=0.19$ by the Student's t-test) was not different between patients with and without troponin elevation. Patients with troponin elevation were older and had a more rapid heart rate, previous history of IHD and $\mathrm{CHF}$, and comorbid AF, renal insufficiency and active cancer than those without troponin elevation. In terms of stroke characteristics, patients with troponin elevation often displayed higher NIHSS score and more cardiogenic embolic subtypes present than other etiologies of stroke compared with those without troponin elevation (Table 1).

\section{Factors affecting troponin elevation}

A multivariable regression analysis revealed previous IHD (odds ratio $[\mathrm{OR}] 2.35,95 \%$ confidence interval $[\mathrm{Cl}]$ 1.49-3.70), CHF (OR 2.05, 95\% Cl 1.31-3.22), comorbid AF (OR 1.52, 95\% Cl 1.04-2.23), active cancer (OR 4.99, 95\% Cl 2.98-8.37), and increased stroke severity (1-point increase in NIHSS score; OR $1.05,95 \% \mathrm{Cl} 1.02-1.08$ ) were identified as factors affecting troponin elevation (Supplementary Table 2).

\section{Cause of death and prognostic value of troponin elevation}

Over a median follow-up period of 33 months (interquartile range, 20-48 months), 331 patients (19.6\%) died. Patients with troponin elevation had a higher risk of death ( $46 \%$ vs. $17 \%$ ) during observation period than those without troponin elevation. Of the time-specific cause of death, stroke-related $(P<0.01)$ and cancer-related $(P<0.01)$ deaths frequently occurred within 1 year after stroke, whereas cardiac-related $(P<0.01)$ death was predominated beyond one year after stroke

Distribution of troponin levels

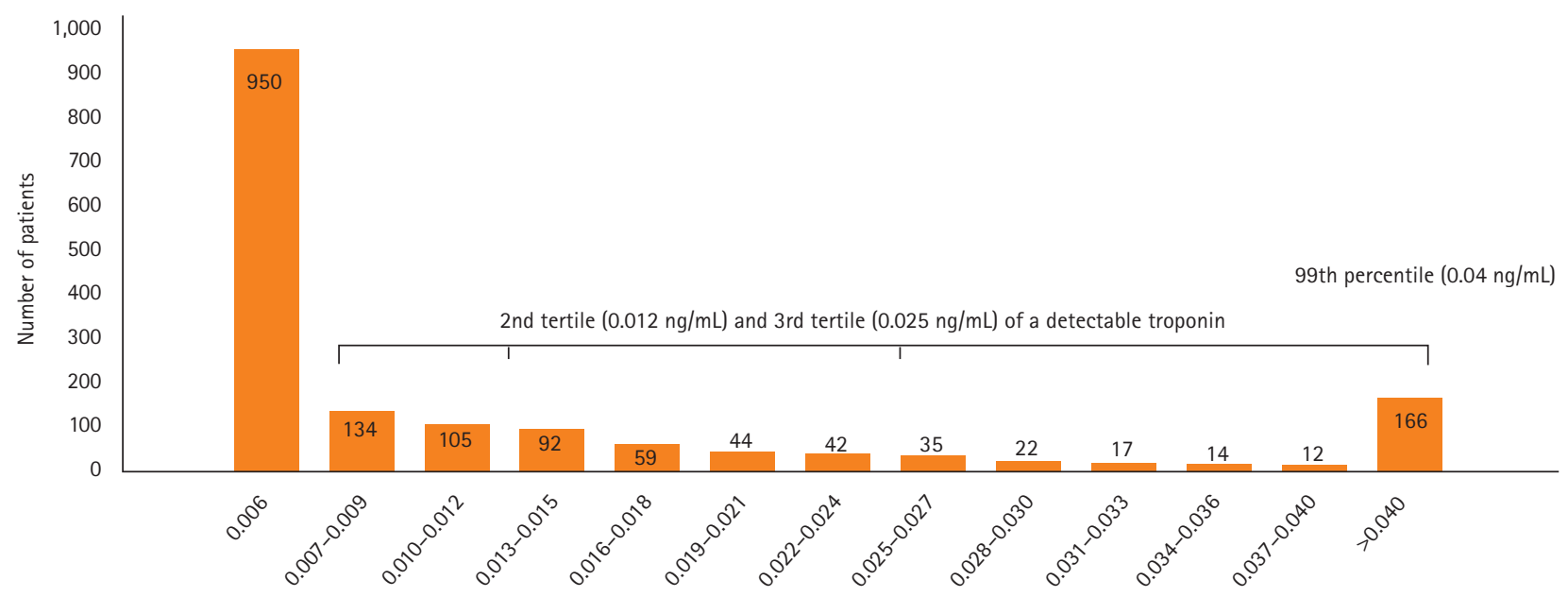

Figure 1. Distribution of serum cardiac troponin I level and different cut-off point for the definition of troponin elevation. 
in patients with troponin elevation than those without troponin elevation (Figure 2). The Kaplan-Meier plot for the longterm survival revealed that troponin elevation was associated with higher mortality and incidence of stroke-, cardiac-, and cancer-related death than those without troponin elevation during the 6-year period (Figure 3).

As shown in Table 2, the risk of overall mortality, stroke-related, cardiac-related, and cancer-related death was significantly higher in the patients with troponin elevation than in patients without troponin elevation using adjusted multivariable analysis using Cox regression method and IPTW method. In addition, using a different definition of troponin elevation based on tertiles of a detectable troponin I level, the overall mortality was still higher in patients with a troponin level exceeding the 3rd tertile of a detectable troponin level than in those without troponin elevation mainly due to an increased risk of stroke- and cardiac-related death. The overall mortality tended to be higher in the patients with a troponin level ex- ceeding the 2 nd tertile of a detectable troponin level than in those without troponin elevation mainly due to an increased risk of cardiac-related death. These trends persisted among the different etiological subgroups even after adjusting for baseline characteristics with the additional inclusion of CRP level (Supplementary Table 3).

\section{Burden of comorbidities and stroke severity in four subgroups according to troponin level}

Prevalence of comorbidities (e.g., included AF, previous IHD, $\mathrm{CHF}$, and renal insufficiency) and median NIHSS score $(4[2,8]$ and $4[2,8]$ vs. $5[2,10]$ vs. $6[3,14] ; P<0.01$ followed by Tukey's posthoc test) was gradually increased with increasing levels of troponin (Figure 4).

\section{Echocardiographic abnormalities in troponin elevation}

Among 1,692 patients, 988 patients (58.4\%) performed trans-
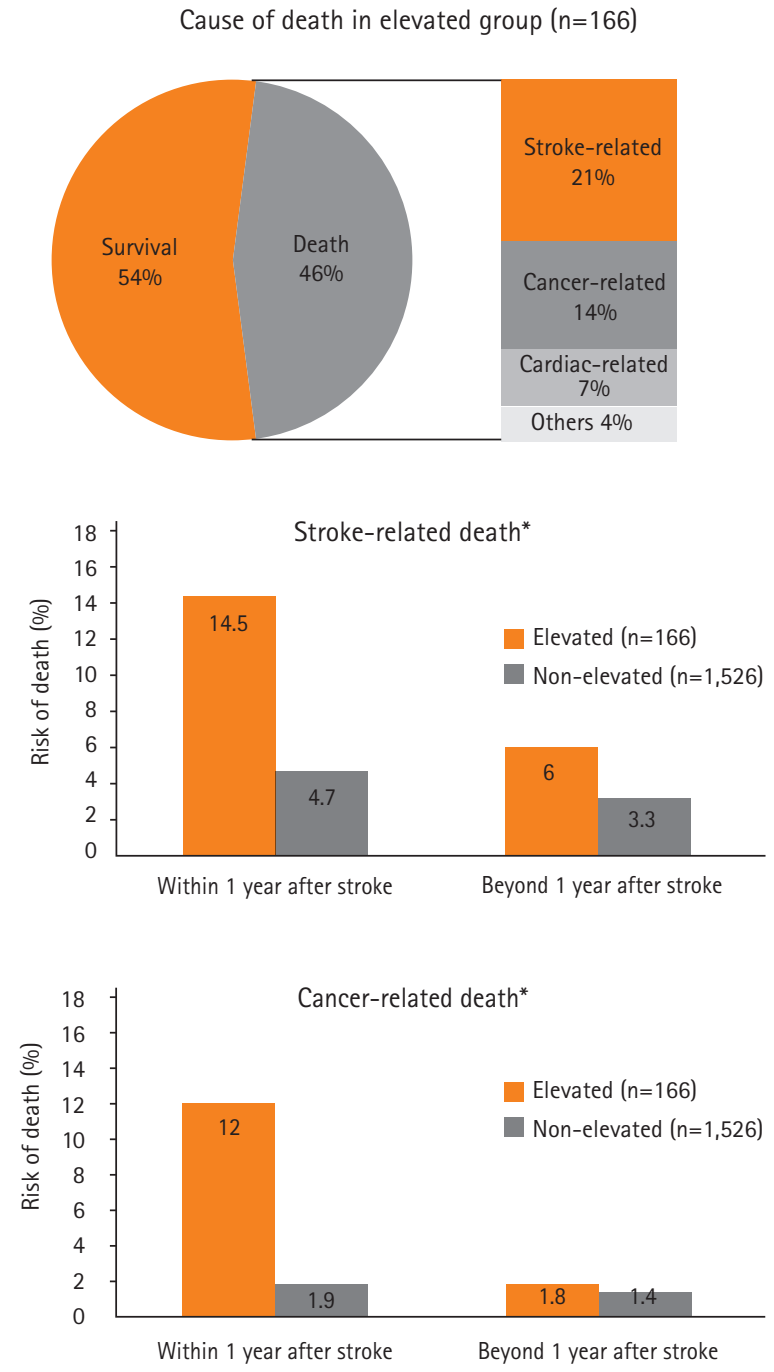

Cause of death in non-elevated group $(n=1,526)$

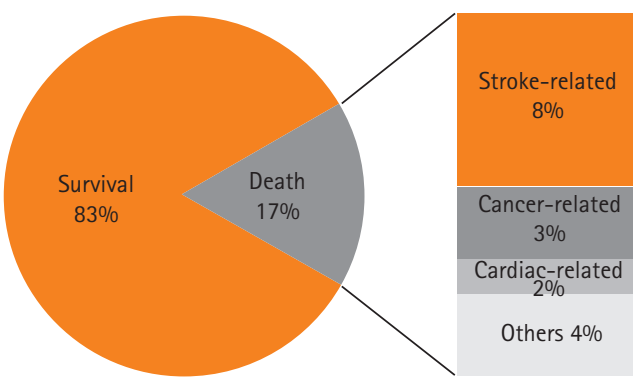

A

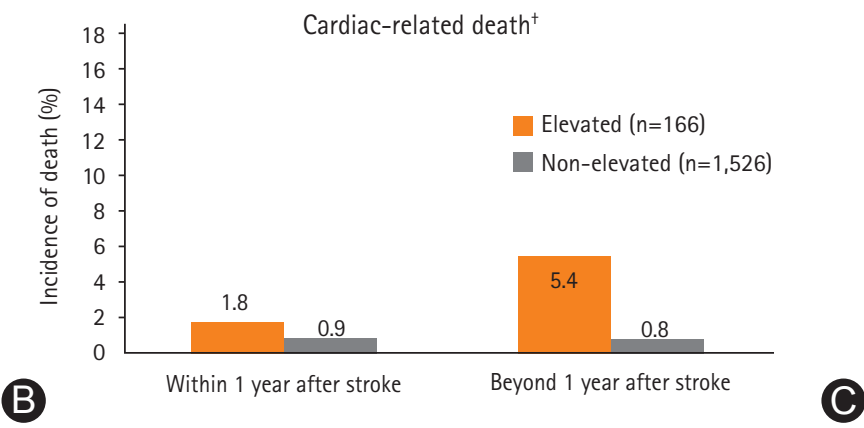

Figure 2. Overall cause of death in elevated and non-elevated troponin groups. Overall cause of death (A) and percentage of stroke-related (B), cardiac-related (C), and cancer-related (D) death within and beyond 1 year after stroke onset in elevated and non-elevated troponin groups. ${ }^{*} P<0.05$ using Chi-square test; ${ }^{\dagger} P<0.05$ using Fisher's exact test. 

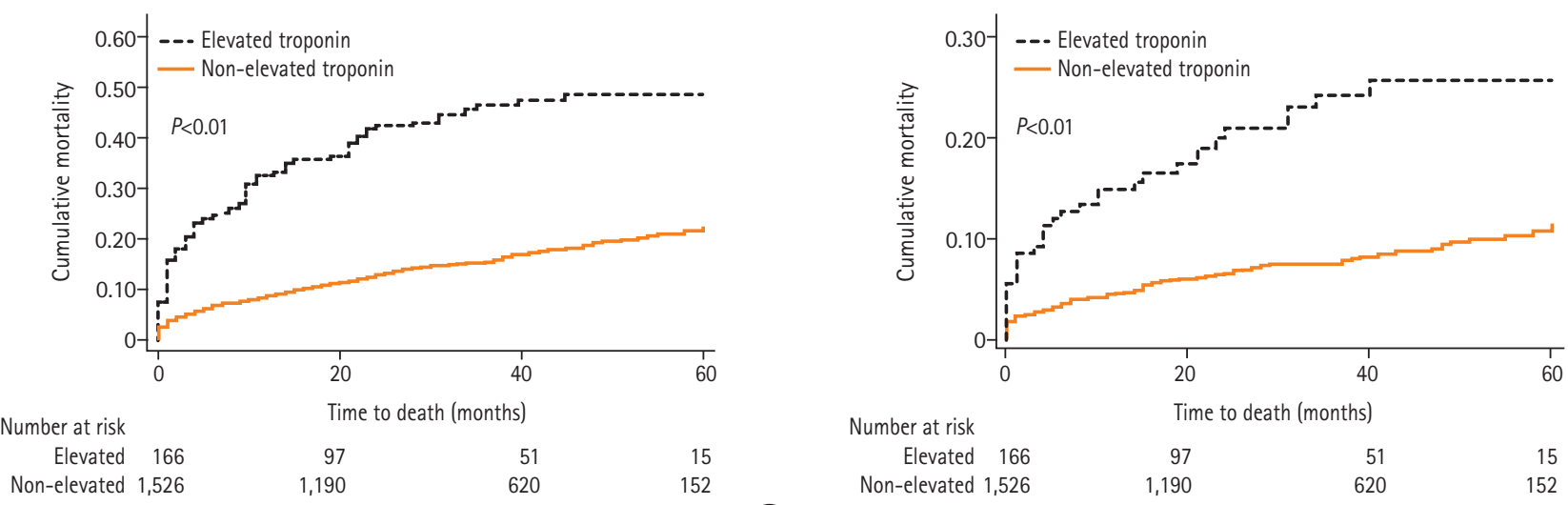

A
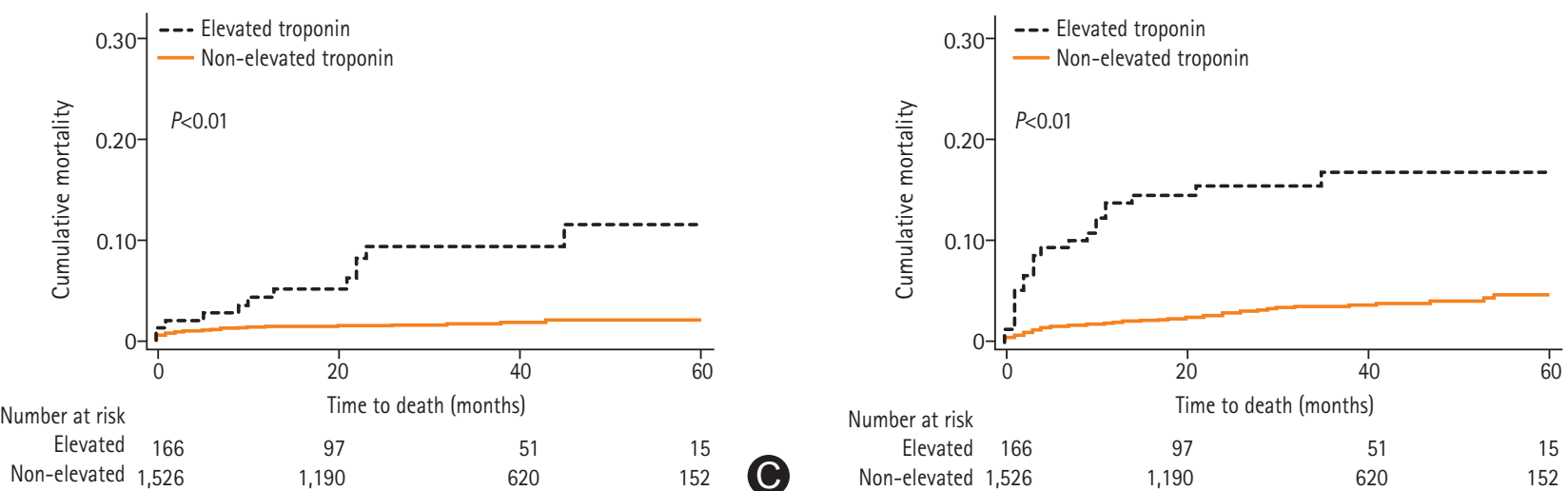

Figure 3. Kaplan-Meier plots in elevated and non-elevated troponin groups. Kaplan-Meier plots of overall mortality (A) and risk of stroke-related (B), cardiac-related (C), and cancer-related (D) deaths in relation to baseline troponin levels. $P$-values are derived using the log-rank test.

thoracic echocardiography (Supplementary Table 4). The overall prevalence of echocardiographic abnormalities including WMA, and reduced $\mathrm{EF}$ and $\mathrm{HM}$ were higher in patients with troponin elevation than those without elevation, and still higher in patients with troponin elevation than those without elevation among patients having a previous history of IHD, CHF, or both (Figure 5).

\section{Discussion}

This study revealed the high prognostic significance of troponin elevation for long-term mortality in patients with acute ischemic stroke. Stroke patients with elevated troponin levels had a higher risk of death (46\% vs. $17 \%$ ) than those without elevated troponin level, even after controlling for multiple factors that might contribute to mortality. We also identified the factors affecting troponin elevation, which could explain the increased risk of stroke-, cardiac-, and cancer-related death in patients with troponin elevation. Furthermore, even after using a lower cut-off point (e.g., the 3 rd tertile $[0.025 \mathrm{ng} / \mathrm{mL}$ ] or the 2nd tertile [0.012 ng/mL] of a detectable troponin) compared to the standard cut-off point (e.g., the 99th percentile [0.040 $\mathrm{ng} / \mathrm{mL}]$ ) to define troponin elevation, the overall mortality was still higher or tended to be higher in patients with troponin elevation than in those without elevation, respectively (Table 2), and still persisted among the different etiological subgroups (e.g., cardioembolic and non-cardioembolic stroke subtype; Supplementary Table 3). Traditionally, in the presence of a clinical history suggestive of acute coronary syndrome, the maximal concentration of cardiac troponin exceeding the 99th percentile of values is considered indicative of myocardial necrosis consistent with myocardial infarction. ${ }^{21}$ However, with the widespread use of more sensitive assays for cardiac troponin in various populations, the proportion of patients with low-level elevations of cardiac troponin below the 99th percentile has increased substantially and these low-level elevations are still associated with future adverse cardiovascular outcomes. ${ }^{22}$ Thus, our results also demonstrate that cardiac troponin values 
Table 2. Unadjusted and adjusted hazard ratios of mortality over 4.5 years in patients with troponin elevation defined by the standard cut-off point or tertiles of a detectable troponin

\begin{tabular}{|c|c|c|c|c|c|c|}
\hline \multirow{2}{*}{ Death } & \multicolumn{2}{|c|}{ Crude } & \multicolumn{2}{|c|}{ Multivariable Cox PH model* } & \multicolumn{2}{|c|}{ Stabilized IPTW } \\
\hline & HR & $95 \% \mathrm{Cl}$ & $H R$ & $95 \% \mathrm{Cl}$ & HR & $95 \% \mathrm{Cl}$ \\
\hline \multicolumn{7}{|c|}{ Troponin elevation (>the 99th percentile of troponin; $0.040 \mathrm{ng} / \mathrm{mL}$ ) } \\
\hline All-cause & 3.33 & $2.58-4.30$ & 1.83 & $1.40-2.40$ & 2.03 & $1.45-2.83$ \\
\hline Stroke-related & 3.06 & $2.09-4.48$ & 1.71 & $1.14-2.55$ & 1.92 & $1.21-3.06$ \\
\hline Cardiac-related & 5.64 & $2.72-11.72$ & 3.17 & $1.49-6.74$ & 3.43 & $1.55-7.60$ \\
\hline Cancer-related & 5.04 & $3.08-8.25$ & 1.98 & $1.14-3.45$ & 2.25 & $1.19-4.25$ \\
\hline \multicolumn{7}{|c|}{ Troponin elevation (>the 3 rd tertile of a detectable troponin; $0.025 \mathrm{ng} / \mathrm{mL}$ ) } \\
\hline All-cause & 2.83 & $2.25-3.58$ & 1.56 & $1.21-2.00$ & N/A & \\
\hline Stroke-related & 2.59 & $1.84-3.66$ & 1.54 & $1.06-2.22$ & $\mathrm{~N} / \mathrm{A}$ & \\
\hline Cardiac-related & 4.82 & $2.39-9.69$ & 2.48 & $1.19-5.13$ & N/A & \\
\hline Cancer-related & 3.43 & $2.13-5.53$ & 1.31 & $0.76-2.26$ & N/A & \\
\hline \multicolumn{7}{|c|}{ Troponin elevation (>the $2 \mathrm{nd}$ tertile of a detectable troponin; $0.012 \mathrm{ng} / \mathrm{mL}$ ) } \\
\hline All-cause & 2.00 & $1.61-2.48$ & 1.22 & $0.97-1.53$ & $\mathrm{~N} / \mathrm{A}$ & \\
\hline Stroke-related & 1.87 & $1.37-2.57$ & 1.14 & $0.82-1.59$ & $\mathrm{~N} / \mathrm{A}$ & \\
\hline Cardiac-related & 4.59 & $2.21-9.53$ & 2.35 & $1.10-5.04$ & $\mathrm{~N} / \mathrm{A}$ & \\
\hline Cancer-related & 1.74 & $1.10-2.77$ & 0.86 & $0.51-1.43$ & $\mathrm{~N} / \mathrm{A}$ & \\
\hline
\end{tabular}

$\mathrm{PH}$, proportional hazards; IPTW, inverse-probability-of-treatment weighting; HR, hazard ratio; $\mathrm{Cl}$, confidence interval; N/A, not applicable.

*Adjustments were made for variables listed in Table 1 except for the stroke subtypes.

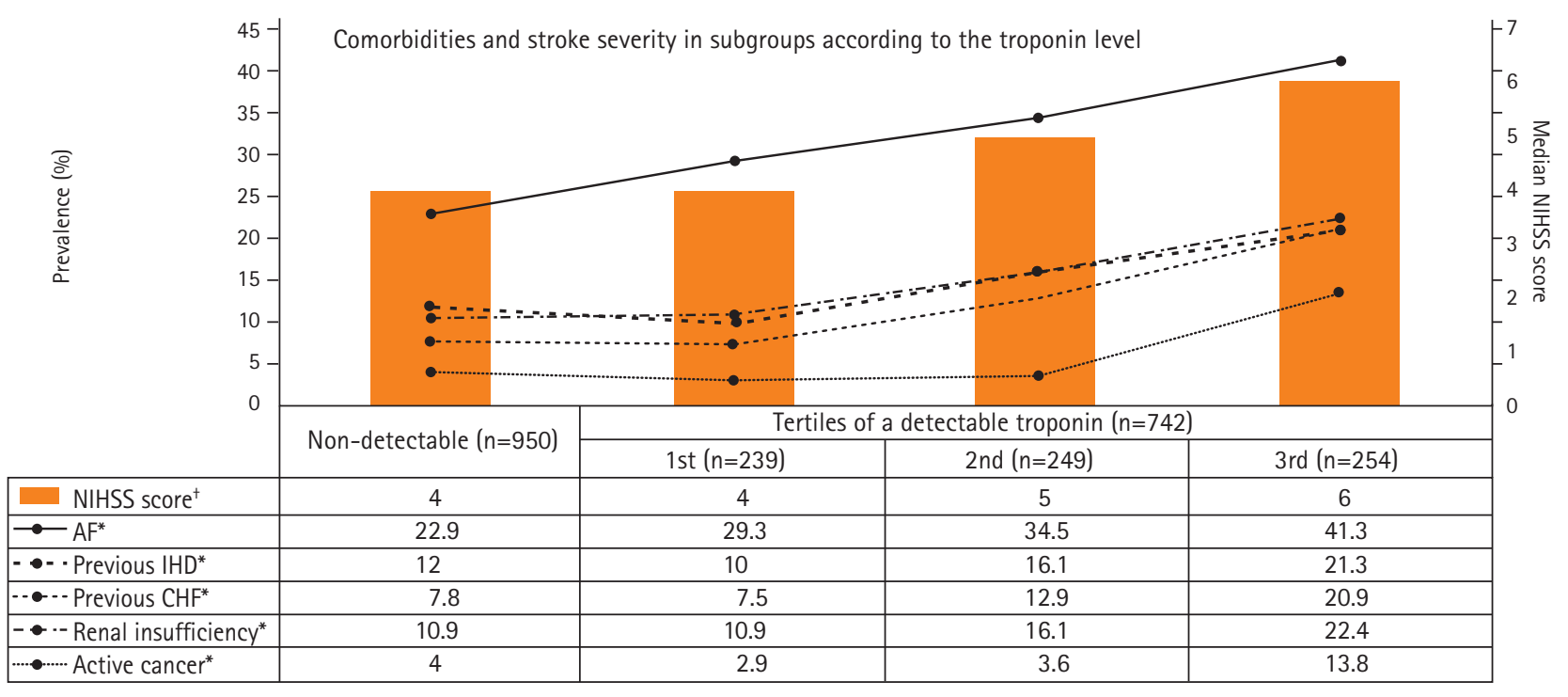

Figure 4. Comorbidities and stroke severity in four subgroups according to the troponin level. Prevalence of cardiac- and non-cardiac comorbidities and median score of NIHSS in four subgroups according to the tertile ranges of a detectable troponin and non-detectable troponin. NIHSS, National Institutes of Health stroke scale; $A F$, atrial fibrillation; IHD, ischemic heart disease; $C H F$, congestive heart failure. ${ }^{*} P<0.05$ using linear-by-linear association $C h i-s q u a r e$ test; ${ }^{\dagger} P<0.05$ using analysis of variance (ANOVA).

at or even below the 99th percentile have a prominent role in the risk prediction of long-term mortality after ischemic stroke.

We showed that troponin elevation was closely associated with increased stroke severity, leading to increased risk of stroke-related death, particularly within 1 year after stroke (Figure 2B). Early mortality in stroke patients depends strongly on the severity of stroke upon hospital admission ${ }^{23}$ and is usually related to cardioembolic stroke because of greater risk of 
Prevalence of echocardiographic abnormalities

Non-elevated $(n=880)$

Elevated $(n=108)$

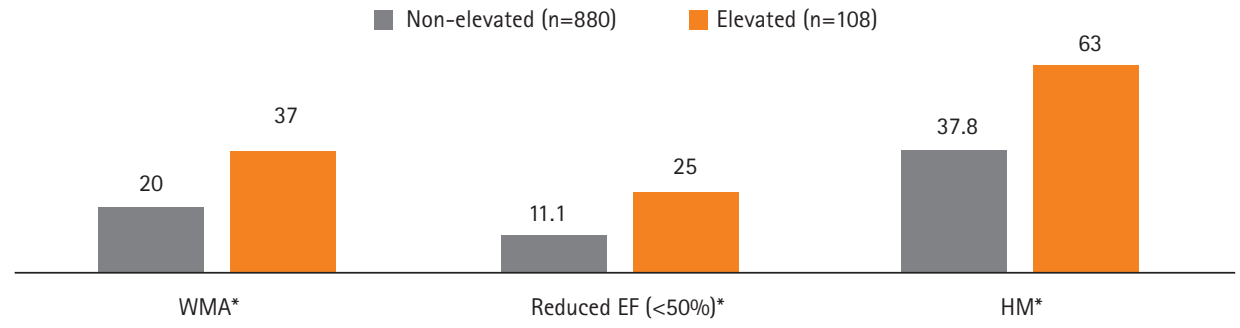

In patients with previous IHD or CHF $(n=290)$

In patients without previous IHD or CHF ( $n=698)$

Non-elevated $(n=644) \quad$ Elevated $(n=54)$
Non-elevated $(n=236) \quad$ Elevated $(n=54)$
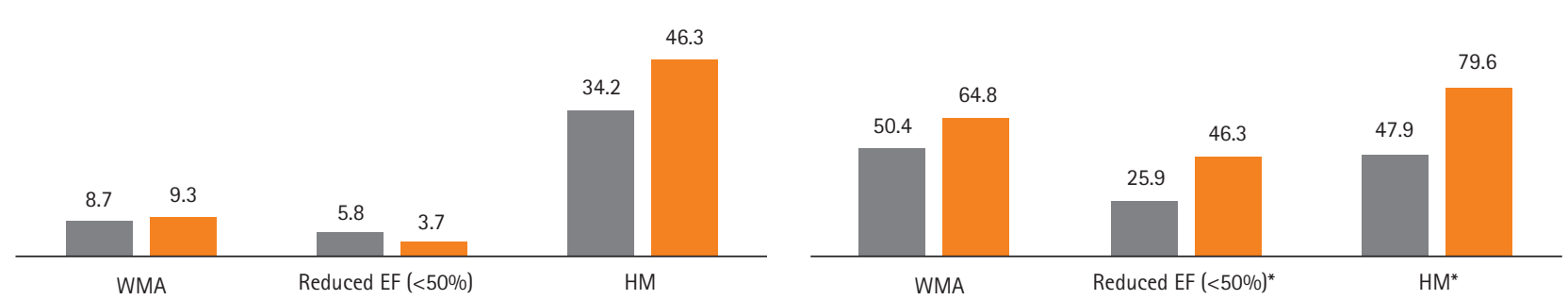

B

Figure 5. Prevalence of echocardiographic abnormalities in elevated and non-elevated troponin group. Prevalence of echocardiographic abnormalities in elevated and non-elevated troponin groups (A) and in subgroups according to the presence of a previous history of IHD, CHF, or both (B). WMA, wall motion abnormalities; $\mathrm{EF}$, ejection fraction; $\mathrm{HM}$, hypertrophic myocardium; IHD, ischemic heart disease; $\mathrm{CHF}$, congestive heart failure. ${ }^{*} P<0.05$ by chi-square test.

baseline severity than other etiologies. ${ }^{24}$ Furthermore, previous literature suggests that troponin elevation can be a marker of stroke severity through the mechanism of neurally mediated myocytolysis, ${ }^{25}$ as well as emboligenic cardiac conditions mainly related to $A \mathrm{~F}^{26}$ Therefore, troponin elevation can reflect stroke severity at baseline and predict future risk of stroke-related death mainly due to cardioembolic stroke. Indeed, the overall mortality in our patients with troponin elevation (46\% during the median of 33 months) was similar to the previous results of cardioembolic stroke (40-60\% of fatality rate within 5 years after ischemic stroke). ${ }^{27}$

We found that troponin elevation was also associated with a previous history of IHD and CHF as well as concomitant $\mathrm{AF}_{\text {, }}$ leading to increased risk of cardiac-related death particularly beyond 1 -year after stroke (Figure $2 \mathrm{C}$ ). Cardiovascular complications are responsible for 2-6\% of total mortalities 3 months after ischemic stroke; ${ }^{28}$ thus, for 1 -year survivors of stroke, cardiac death is the most common cause of death during the first 5 years. ${ }^{29}$ In our study, although the rate of overall cardiac-related deaths $(0.7 \%$ per year; 32 of 1,692 cardiac deaths for median of 33 months) was rather lower than that of previous meta-analysis (the annual risk of non-stroke vascular death; 2.2\% [95\% Cl 1.7-2.7] in patients with ischemic stroke during the period of 1980 to 2005) ${ }_{1}^{30}$ the rate of cardiac-related death in patients with troponin elevation (4.4\% per year; 22 of 166 deaths) was still high despite the initial exclusion of patients with suspicion of acute coronary syndrome. Furthermore, patients with troponin elevation had more severely deteriorated structural/functional cardiac abnormalities diagnosed by echocardiography than those without troponin elevation even with a previous history of IHD, CHF or both (Figure 5B). Thus, the risk of stroke- and cardiac-related death in patients with troponin elevation could be further increased beyond known or undetected underlying comorbidities.

Interestingly, non-vascular etiology such as cancer-related death was also six times higher in patients with troponin elevation, particularly within 1 year after stroke (Figure 2D). Cancer is known as one of the main causes of mortality in survivors after stroke because the incidence of cancer increases with increasing age. ${ }^{31}$ Furthermore, cancer is a risk factor for stroke because cancer and its treatments cause alterations in platelet, coagulation, and endothelial functions, which in turn can lead to thrombosis or hemorrhage. ${ }^{32}$ Although available data are scarce, several studies have shown that the troponin elevation or myocardial strain measured by echocardiography correlates with the risk of high-dose chemotherapy-induced cardiotoxicity. ${ }^{33}$ Thus, 
future studies are required to focus on the cellular level of myocytic damage and discriminate whether those elevations are due to chemotherapy (e.g., type and duration of treatment) or cancer itself (e.g., type and stage of cancer and the presence of metastasis or hypercoagulable state). ${ }^{34}$

Although our findings show that troponin elevation can indicate various vascular and non-vascular problems and consequently predict future vascular and non-vascular death, the clinical value of measuring troponin is still limited due to an additional diagnostic challenge to discriminate various conditions for troponin elevation. ${ }^{35}$ Currently, we proposed a concept that synergistic effect on troponin elevation in a combination of the cardiac vulnerability (e.g., hypertrophic or damaged myocardium by ischemia, and possibly tachycardia) to cerebrogenic stress (e.g., by severe neurological deficits or insular cortical lesion) could be a practical interpretation of troponin elevation in stroke patients. ${ }^{36}$ In our study, the median score of NIHSS and prevalence of comorbidities (e.g., mainly due to a gradual increase in cardiac-comorbidities including $A F_{1} I H D_{\text {, }}$ and CHF) was gradually increased in a dose-dependent manner with increase in four levels of troponin (Figure 4). Therefore, for the prediction of cardiac-related death, the cut-off point for troponin level could be lowered below the 99th percentile because of the gradual increase in cardiac vulnerability in parallel with a gradual increase in troponin from the very low-level elevations. Otherwise, for the prediction of stroke- or cancer-related death, the 99th percentile of troponin would be an appropriate cut-off value because of the additive neurogenic stress on vulnerable cardiac status or additional myocardial damage/dysfunction induced by cancer- or chemotherapy-related insult, which usually leads to further increase in the troponin level compared to isolated cardiac disease.

There are some limitations of our study. First, the design of the study was retrospective, and the study was performed at a single center. Second, we could not show non-fatal major adverse cardiac events because we reconfirmed the rate of longterm mortality using the data obtained from the National Death Certificate system for the investigation of precise rate of mortality. Third, we were unable to perform intensive cardiac investigations comprising serial troponin and ECG testing and coronary angiography. Thus, we could not identify the patients with subclinical cardiac events like as non-ST segment elevation myocardial infarction or stress-induced cardiomyopathy during hospitalization leading to underestimation of the frequency of troponin elevation and cardiac comorbidities. Thus, we are currently conducting a prospective observational study with serial troponin and ECG tests (for detection of newly de- veloped cardiac events), and serum B-type natriuretic peptide (e.g., BNP for identifying heart failure) and D-dimer/Fibrinogen (for identifying cancer activity) in acute ischemic stroke patients (Clinical implications of elevated cardiac troponin-I elevation in acute stroke patients; KCT0000682; https://cris.nih. go.kr/cris). Future studies should focus on adjusting for covariables with possible impact on vascular or cancer-related mortality including other laboratory parameters and detailed information of cancer state to reduce possible confounding effects.

\section{Conclusions}

Troponin elevation in ischemic stroke was prevalent and associated with long-term mortality independent of other known risk factors. The etiology of troponin elevation and the cause of death in stroke patients with troponin elevation were correlated; this could explain the reasons for increased risk of strokeand cancer-related death in the first year and cardiac-related death in the later period. Furthermore, even after using a lower cut-off value to define troponin elevation compared to the standard value, the overall mortality was higher in patients with troponin elevation than in those without elevation. Thus, the measurement of troponin level could be helpful in patients with stroke for identifying the underlying cardiac and non-cardiac problems and useful for risk estimation for long-term mortality during the post-stroke period.

\section{Acknowledgements}

This study was supported by a grant from the Korea Healthcare Technology R\&D Project, Ministry of Health and Welfare Republic of Korea (HI10C2O20 and HI14C1731).

\section{Supplementary materials}

Supplementary materials related to this article can be found online at https://doi.org/10.5853/jos.2016.01942.

\section{References}

1. Thygesen $K$, Alpert JS, Jaffe AS, Simoons ML, Chaitman BR, White HD, et al. Third universal definition of myocardial infarction. J Am Coll Cardiol 2012;60:1581-1598.

2. Popp RL. Troponin: messenger or actor? J Am Coll Cardiol 2013;61:611-614.

3. Fure B, Bruun Wyller T, Thommessen B. Electrocardiographic and troponin $\mathrm{T}$ changes in acute ischaemic stroke. J Intern 
Med 2006;259:592-597.

4. Jensen JK, Kristensen SR, Bak S, Atar D, Høilund-Carlsen PF, Mickley $\mathrm{H}$. Frequency and significance of troponin T elevation in acute ischemic stroke. Am J Cardiol 2007;99:108-112.

5. Kerr G, Ray G, Wu O, Stott DJ, Langhorne P. Elevated troponin after stroke: a systematic review. Cerebrovasc Dis 2009; 28:220-226.

6. Di Angelantonio E, Fiorelli M, Toni D, Sacchetti ML, Lorenzano $S$, Falcou $A$, et al. Prognostic significance of admission levels of troponin I in patients with acute ischaemic stroke. $J$ Neurol Neurosurg Psychiatry 2005;76:76-81.

7. Scheitz JF, Endres M, Mochmann HC, Audebert HJ, Nolte $\mathrm{CH}$. Frequency, determinants and outcome of elevated troponin in acute ischemic stroke patients. Int J Cardiol 2012;157:239242.

8. Palma JA, Benarroch EE. Neural control of the heart: recent concepts and clinical correlations. Neurology 2014;83:261271.

9. Thygesen K, Mair J, Giannitsis E, Mueller C, Lindahl B, Blankenberg $S$, et al. How to use high-sensitivity cardiac troponins in acute cardiac care. Eur Heart J 2012;33:2252-2257.

10. Adams HP Jr, del Zoppo G, Alberts MJ, Bhatt DL, Brass L, FurIan $A$, et al. Guidelines for the early management of adults with ischemic stroke: a guideline from the American Heart Association/American Stroke Association Stroke Council, Clinical Cardiology Council, Cardiovascular Radiology and Intervention Council, and the Atherosclerotic Peripheral Vascular Disease and Quality of Care Outcomes in Research Interdisciplinary Working Groups: The American Academy of Neurology affirms the value of this guideline as an educational tool for neurologists. Circulation 2007;115:e478-e534.

11. Brott T, Adams HP Jr, Olinger CP, Marler JR, Barsan WG, Biller $J$, et al. Measurements of acute cerebral infarction: a clinical examination scale. Stroke 1989;20:864-870.

12. Hong KS, Ko SB, Yu KH, Jung C, Park SO, Kim BM, et al. Update of the Korean clinical practice guidelines for endovascular recanalization therapy in patients with acute ischemic stroke. J Stroke 2016;18:102-113.

13. Adams HP Jr, Bendixen BH, Kappelle $\sqcup$, Biller J, Love BB, Gordon DL, et al. Classification of subtype of acute ischemic stroke. Definitions for use in a multicenter clinical trial. TOAST. Trial of org 10172 in acute stroke treatment. Stroke 1993;24:35-41.

14. Prineas RJ, Crow RS, Zhang ZM. The Minnesota Code Manual of Electrocardiographic Findings. 2nd ed. London: Springer, 2010; XIII, 328.

15. Lang RM, Bierig $M$, Devereux RB, Flachskampf FA, Foster $E_{1}$
Pellikka PA, et al. Recommendations for chamber quantification: a report from the American Society of Echocardiography's Guidelines and Standards Committee and the Chamber Quantification Writing Group, developed in conjunction with the European Association of Echocardiography, a branch of the European Society of Cardiology. J Am Soc Echocardiogr 2005;18:1440-1463.

16. de Simone G, Daniels SR, Kimball TR, Roman MJ, Romano C, Chinali $\mathrm{M}$, et al. Evaluation of concentric left ventricular geometry in humans: evidence for age-related systematic underestimation. Hypertension 2005;45:64-68.

17. World Health Organization. International statistical classification of diseases and related health problems. 10th ed. Geneva: WHO, 1992.

18. Robins JM, Hernan MA, Brumback B. Marginal structural models and causal inference in epidemiology. Epidemiology 2000;11:550-560.

19. Hernán MA, Brumback $B$, Robins JM. Marginal structural models to estimate the causal effect of zidovudine on the survival of HIV-positive men. Epidemiology 2000;11:561570.

20. Brookhart MA, Wyss R, Layton JB, Stürmer T. Propensity score methods for confounding control in nonexperimental research. Circ Cardiovasc Qual Outcomes 2013;6:604-611.

21. Thygesen $K$, Alpert JS, White HD. Universal definition of myocardial infarction. J Am Coll Cardiol 2007;50:2173-2195.

22. Keller T, Ojeda F, Zeller T, Wild PS, Tzikas S, Sinning CR, et al. Defining a reference population to determine the 99th percentile of a contemporary sensitive cardiac troponin I assay. Int J Cardiol 2013;167:1423-1429.

23. Vernino S, Brown RD Jr, Sejvar JJ, Sicks JD, Petty GW, O'Fallon WM. Cause-specific mortality after first cerebral infarction: a population-based study. Stroke 2003;34:1828-1832.

24. Lamassa M, Di Carlo A, Pracucci G, Basile AM, Trefoloni G, Vanni $P$, et al. Characteristics, outcome, and care of stroke associated with atrial fibrillation in Europe: data from a multicenter multinational hospital-based registry (The European Community Stroke Project). Stroke 2001;32:392-398.

25. Song HS, Back JH, Jin DK, Chung PW, Moon HS, Suh BC, et al. Cardiac troponin T elevation after stroke: relationships between elevated serum troponin $\mathrm{T}$, stroke location, and prognosis. J Clin Neurol 2008;4:75-83.

26. van den Bos EJ, Constantinescu AA, van Domburg RT, Akin S, Jordaens $\sqcup$, Kofflard MJ. Minor elevations in troponin I are associated with mortality and adverse cardiac events in patients with atrial fibrillation. Eur Heart J 2011;32:611-617.

27. Fang MC, Go AS, Chang Y, Borowsky LH, Pomernacki NK, 
Udaltsova $\mathrm{N}$, et al. Long-term survival after ischemic stroke in patients with atrial fibrillation. Neurology 2014;82:10331037.

28. Touzé E, Varenne O, Chatellier G, Peyrard S, Rothwell PM, Mas JL. Risk of myocardial infarction and vascular death after transient ischemic attack and ischemic stroke: a systematic review and meta-analysis. Stroke 2005;36:2748-2755.

29. Hardie K, Hankey GJ, Jamrozik K, Broadhurst RJ, Anderson C. Ten-year survival after first-ever stroke in the perth community stroke study. Stroke 2003;34:1842-1846.

30. Hardie K, Jamrozik K, Hankey GJ, Broadhurst RJ, Anderson C. Trends in five-year survival and risk of recurrent stroke after first-ever stroke in the Perth Community Stroke Study. Cerebrovasc Dis 2005;19:179-185.

31. Brønnum-Hansen $H$, Davidsen $M$, Thorvaldsen $P$; Danish MONICA Study Group. Long-term survival and causes of death after stroke. Stroke 2001;32:2131-2136.
32. Caine GJ, Stonelake PS, Lip GY, Kehoe ST. The hypercoagulable state of malignancy: pathogenesis and current debate. Neoplasia 2002;4:465-473.

33. Cardinale D, Sandri MT, Colombo A, Colombo N, Boeri M, Lamantia $G$, et al. Prognostic value of troponin I in cardiac risk stratification of cancer patients undergoing high-dose chemotherapy. Circulation 2004;109:2749-2754.

34. Cestari DM, Weine DM, Panageas KS, Segal AZ, DeAngelis LM. Stroke in patients with cancer: incidence and etiology. Neurology 2004;62:2025-2030.

35. Mahajan VS, Jarolim P. How to interpret elevated cardiac troponin levels. Circulation 2011;124:2350-2354.

36. Ahn SH, Kim YH, Shin CH, Lee JS, Kim BJ, Kim YJ, et al. Cardiac vulnerability to cerebrogenic stress as a possible cause of troponin elevation in stroke. J Am Heart Assoc 2016; 5:e004135. 
Supplementary Table 1. Baseline characteristics of imbalance check result after stabilized IPTW

\begin{tabular}{|c|c|c|c|}
\hline \multirow{2}{*}{ Variable } & \multicolumn{2}{|c|}{ After stabilized IPTW cardiac troponin I } & \multirow{2}{*}{$P^{*}$} \\
\hline & Elevated & Non-elevated & \\
\hline Age (years) & $65.6 \pm 14.7$ & $66.0 \pm 12.3$ & 0.78 \\
\hline Male & $103(62.7)$ & $934(61.1)$ & 0.73 \\
\hline Heart rate (beats a minute) & $79.3 \pm 18.6$ & $77.7 \pm 18.0$ & 0.34 \\
\hline \multicolumn{4}{|l|}{ Medical history } \\
\hline Hypertension & $106(64.7)$ & $973(63.7)$ & 0.83 \\
\hline Diabetes mellitus & $38(23.5)$ & $385(25.2)$ & 0.68 \\
\hline Hyperlipidemia & $38(23.3)$ & $349(22.8)$ & 0.91 \\
\hline Current smoking & $54(33.2)$ & $467(30.6)$ & 0.56 \\
\hline \multicolumn{4}{|l|}{ Comorbidities } \\
\hline Previous stroke & $45(27.5)$ & $398(26.0)$ & 0.74 \\
\hline Previous ischemic heart disease & $22(13.2)$ & $210(13.7)$ & 0.83 \\
\hline Previous congestive heart failure & $19(11.5)$ & $162(10.6)$ & 0.70 \\
\hline Atrial fibrillation & $47(29.0)$ & $435(28.4)$ & 0.88 \\
\hline Renal insufficiency & $23(14.2)$ & $206(13.5)$ & 0.80 \\
\hline Active cancer & $11(7.0)$ & $99(6.4)$ & 0.74 \\
\hline Reperfusion therapy & $24(15.0)$ & $185(12.1)$ & 0.43 \\
\hline NIHSS score & $6.2 \pm 6.1$ & $6.4 \pm 6.1$ & 0.80 \\
\hline
\end{tabular}

Values are presented mean \pm standard deviation, or number (\%).

IPTW, inverse-probability-of-treatment weighting; NIHSS, National Institutes of Health stroke scale.

${ }^{*} P$-values are calculated by generalized estimating equation method. 
Supplementary Table 2. Factors affecting troponin elevation

\begin{tabular}{|c|c|c|c|c|}
\hline \multirow{2}{*}{ Variables } & \multicolumn{2}{|c|}{ Unadjusted } & \multicolumn{2}{|c|}{ Adjusted* } \\
\hline & $\mathrm{OR}$ & $95 \% \mathrm{Cl}$ & $\mathrm{OR}$ & $95 \% \mathrm{Cl}$ \\
\hline Age, per 1-year increase & 1.02 & $1.01-1.03$ & 1.01 & $0.99-1.02$ \\
\hline Male & 0.88 & $0.64-1.22$ & 1.01 & $0.69-1.49$ \\
\hline Heart rate, per 10-beats increase & 1.10 & $1.01-1.20$ & 1.09 & $1.00-1.18$ \\
\hline Hypertension & 1.21 & $0.86-1.70$ & 1.15 & $0.78-1.67$ \\
\hline Diabetes mellitus & 0.83 & $0.57-1.22$ & 0.80 & $0.53-1.21$ \\
\hline Hyperlipidemia & 0.86 & $0.58-1.28$ & 0.72 & $0.46-1.12$ \\
\hline Current smoking & 0.80 & $0.56-1.15$ & 1.04 & $0.68-1.61$ \\
\hline Previous stroke & 1.00 & $0.69-1.44$ & 0.88 & $0.59-1.30$ \\
\hline Previous ischemic heart disease & 2.04 & $1.38-3.02$ & 2.35 & $1.49-3.70$ \\
\hline Previous congestive heart failure & 2.60 & $1.73-3.93$ & 2.05 & $1.31-3.22$ \\
\hline Atrial fibrillation & 1.99 & $1.43-2.77$ & 1.52 & $1.04-2.23$ \\
\hline Renal insufficiency & 1.78 & $1.19-2.67$ & 1.31 & $0.83-2.07$ \\
\hline Active cancer & 4.31 & $2.65-7.03$ & 4.99 & $2.98-8.37$ \\
\hline Reperfusion therapy & 0.93 & $0.56-1.54$ & 0.63 & $0.37-1.08$ \\
\hline NIHSS score, per 1-point increase & 1.06 & $1.04-1.09$ & 1.05 & $1.02-1.08$ \\
\hline
\end{tabular}

$\mathrm{OR}$, odds ratio; $\mathrm{Cl}$, confidence interval; NIHSS, National Institutes of Health Stroke Scale.

*Adjustments were made for variables listed in Table 1 except for the stroke subtypes. 
Supplementary Table 3. Unadjusted and adjusted hazard ratios of mortality over 4.5 years in patients with troponin elevation defined by the standard cutoff point or tertiles of a detectable troponin according to the presumed etiologies of stroke

\begin{tabular}{|c|c|c|c|c|c|c|c|c|}
\hline \multirow{4}{*}{ Death } & \multicolumn{8}{|c|}{ Classified into the presumed etiologies of stroke } \\
\hline & \multicolumn{4}{|c|}{ Stroke due to cardioembolic causes } & \multicolumn{4}{|c|}{ Stroke due to non-cardioembolic causes } \\
\hline & \multicolumn{2}{|c|}{ Crude } & \multicolumn{2}{|c|}{ Multivariable Cox $\mathrm{PH}_{\text {model }}{ }^{*}$} & \multicolumn{2}{|c|}{ Crude } & \multicolumn{2}{|c|}{ Multivariable Cox PH model } \\
\hline & $\mathrm{HR}$ & $95 \% \mathrm{Cl}$ & $\mathrm{HR}$ & $95 \% \mathrm{Cl}$ & $\mathrm{HR}$ & $95 \% \mathrm{Cl}$ & $H R$ & $95 \% \mathrm{Cl}$ \\
\hline \multicolumn{9}{|c|}{ Troponin elevation (>the 99 percentile of troponin; $0.040 \mathrm{ng} / \mathrm{mL}$ ) } \\
\hline All-cause & 1.81 & $1.22-2.69$ & 1.86 & $1.23-2.80$ & 4.62 & $3.29-6.47$ & 1.83 & $1.25-2.68$ \\
\hline Stroke-related & 1.57 & $0.91-2.73$ & 1.42 & $0.78-2.57$ & 4.72 & $2.82-7.92$ & 2.31 & $1.28-4.15$ \\
\hline Cardiac-related & 3.44 & $1.52-7.79$ & 3.90 & $1.62-9.38$ & 6.15 & $1.19-31.78$ & 4.55 & $0.68-30.25$ \\
\hline Cancer-related & 1.78 & $0.37-8.59$ & 6.24 & $0.68-55.78$ & 7.18 & $4.26-12.10$ & 1.72 & $0.95-3.12$ \\
\hline \multicolumn{9}{|c|}{ Troponin elevation (>the $3 \mathrm{rd}$ tertile of a detectable troponin; $0.025 \mathrm{ng} / \mathrm{mL}$ ) } \\
\hline All-cause & 2.09 & $1.15-3.02$ & 2.08 & $1.42-3.06$ & 4.20 & $3.02-5.85$ & 1.61 & $1.09-2.38$ \\
\hline Stroke-related & 1.93 & $1.17-3.18$ & 1.79 & $1.05-3.07$ & 4.22 & $2.54-7.01$ & 2.26 & $1.27-4.02$ \\
\hline Cardiac-related & 3.47 & $1.56-7.73$ & 3.72 & $1.58-8.79$ & 5.17 & $1.00-26.69$ & 3.92 & $0.60-25.63$ \\
\hline Cancer-related & 1.51 & $0.31-7.30$ & 2.69 & $0.31-23.38$ & 6.07 & $3.60-10.22$ & 1.31 & $0.72-2.39$ \\
\hline \multicolumn{9}{|c|}{ Troponin elevation (>the $2 \mathrm{nd}$ tertile of a detectable troponin; $0.012 \mathrm{ng} / \mathrm{mL}$ ) } \\
\hline All-cause & 1.47 & $1.05-2.05$ & 1.21 & $0.85-1.71$ & 2.12 & $1.59-2.83$ & 1.20 & $0.88-1.64$ \\
\hline Stroke-related & 1.75 & $1.08-2.81$ & 1.31 & $0.83-2.04$ & 2.06 & $1.32-3.23$ & 1.38 & $0.86-2.24$ \\
\hline Cardiac-related & 3.23 & $1.39-7.51$ & 2.28 & $0.93-5.57$ & 3.86 & $0.86-17.24$ & 4.06 & $0.78-21.22$ \\
\hline Cancer-related & 0.62 & $0.15-2.53$ & 0.28 & $0.04-1.89$ & 2.26 & $1.38-3.69$ & 0.89 & $0.51-1.54$ \\
\hline
\end{tabular}

$\mathrm{HR}$, hazard ratio; $\mathrm{Cl}$, confidence interval.

${ }^{*}$ Adjustments were made for variables listed in Table 1 except for the stroke subtypes, and level of C-reactive protein was additionally included into the model. 
Supplementary Table 4. Comparison of clinical variables between patients with or without echocardiography

\begin{tabular}{|c|c|c|c|}
\hline \multirow{2}{*}{ Variable } & \multicolumn{2}{|c|}{ Echocardiography } & \multirow{2}{*}{$P^{*}$} \\
\hline & Performed $(n=988)$ & Not-performed $(n=704)$ & \\
\hline Troponin elevation & $108(10.9)$ & $58(8.2)$ & 0.07 \\
\hline Age (years) & $65.8 \pm 12.5$ & $65.8 \pm 12.5$ & 0.49 \\
\hline Male & $607(61.4)$ & $427(60.7)$ & 0.74 \\
\hline Heart rate (beats a minute) & $76.8 \pm 17.6$ & $78.8 \pm 18.4$ & 0.03 \\
\hline \multicolumn{4}{|l|}{ Medical history } \\
\hline Hypertension & $612(61.9)$ & $464(65.9)$ & 0.10 \\
\hline Diabetes mellitus & $249(25.2)$ & $179(25.4)$ & 0.92 \\
\hline Hyperlipidemia & $230(23.3)$ & $156(22.2)$ & 0.59 \\
\hline Current smoking & $295(29.9)$ & $223(31.7)$ & 0.42 \\
\hline \multicolumn{4}{|l|}{ Comorbidities } \\
\hline Previous stroke & $235(23.8)$ & $204(29.0)$ & 0.02 \\
\hline Previous ischemic heart disease & $177(17.9)$ & $55(7.8)$ & $<0.01$ \\
\hline Previous congestive heart failure & $419(15.1)$ & $28(4.0)$ & $<0.01$ \\
\hline Atrial fibrillation & 377 (38.2) & $102(14.5)$ & $<0.01$ \\
\hline Renal insufficiency & $140(14.2)$ & $87(12.4)$ & 0.28 \\
\hline Active cancer & $62(6.3)$ & $27(3.8)$ & 0.03 \\
\hline Reperfusion therapy & $153(15.5)$ & $52(7.4)$ & $<0.01$ \\
\hline NIHSS score & $5[2,10]$ & $4[2,8]$ & 0.15 \\
\hline Stroke subtypes & & & $<0.01$ \\
\hline Large artery atherosclerosis & $286(28.9)$ & $229(32.5)$ & \\
\hline Cardiogenic embolism & $398(40.3)$ & 96 (13.6) & \\
\hline Small vessel disease & $114(11.5)$ & $256(36.4)$ & \\
\hline Undetermined etiology & $140(14.2)$ & $86(12.2)$ & \\
\hline Other etiology & $50(5.1)$ & 37 (5.3) & \\
\hline
\end{tabular}

Values are presented as mean \pm standard deviation, median [interquartile range] or number (\%). NIHSS, National Institutes of Health Stroke Scale.

${ }^{*} P$-values are calculated by Pearson chi-square test or Student's t-test as appropriate. 